\title{
Table of Statutes
}

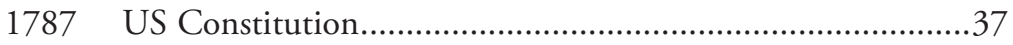

Fourteenth Amendment ......................................................36

1964 Succession (Scotland) Act (c 41) ..........................................182

1967 Abortion Act (c 87)

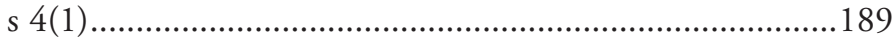

1968 Social Work (Scotland) Act (c 49) ........................................200

1972 European Communities Act (c 68)

s 2(2) ........................................................................

1973 Matrimonial Causes Act (c 18)

Pt II

1974 Rehabilitation of Offenders Act (c 53)

$71,202,237,238,239,241$

s 3

237, 239

s5

238

1975 Inheritance (Provision for Family and Dependants) Act (c 63)

Sex Discrimination Act (c 65)

$53,115,116$

1976 Damages (Scotland) Act (c 13)

$$
63,64,67,85,88,149,150,151
$$

s $1(4 \mathrm{~A})$

s $10(2)$ 151,152

Sch 1 ..... $149,150,151,152$

Property (Relationships) Act (New Zealand) 210,211 s $2 \mathrm{D}$ 210

(2). $.210,212$

1977 Marriage (Scotland) Act (c 15)................ 100, 148, 150, 245 s $5(4)(\mathrm{e})$ 245

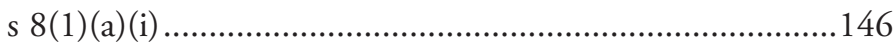
s 17 190 
s $20 \mathrm{~A}$ 261 101

s $23 \mathrm{~A}$ .99

Rent Act (c 42) 86

1978 Adoption (Scotland) Act (c 28) $50,78,134,166$ s 16 165 s 18 .78 (7) $.75,77$ s 20 136

s $50 \mathrm{~A}$. 20 s 65(1) 75

1980 Education (Scotland) Act (c 44) s 1

1981 Matrimonial Homes (Family Protection) (Scotland) Act (c 59) $88,127,130,260$

1983 Solvent Abuse (Scotland) Act (c 33) .................................201

1984 Matrimonial and Family Proceedings Act (c 42) ................155 Rent (Scotland) Act (c 58)

1985 Family Law (Scotland)

Act (c 37)......128, 155, 156, 157, 158, 159, 160, 174, 219 s 8 130,158

s 9 $130,156,157,159$

(1) 158

(a) 158,176 $158,159,160,213$

(c) 158, 207, 208 (d) $156,158,159$ (2)

s 16 221,222

s 24 219

s 25 129

s 26 129

s $27(1)$ 158

Child Abduction and Custody Act (c 60) ..........................171

1986 Law Reform (Parent and Child) (Scotland) Act (c 9) $41,42,180$ s 3(2)

s 5

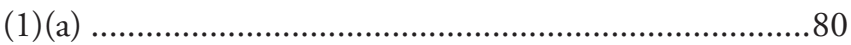

Marriage (Prohibited Degrees of Relationship) Act (c 16)...104 
Incest and Related Offences (Scotland) Act (c 36) 201

Legal Aid (Scotland) Act (c 47)

Family Law Act (c 55)

s 41

1988 Local Government Act (c 9)

s 28 .....

1989 Children Act (c 41)

s 20

1990 Human Fertilisation and Embryology Act (c 37) ...................80, 82, 83, 84, 179, 180, 205, 206 s $13(5)$. $179,180,182,205,206$ s 27 $.80,84$ ss $27-29$. .79 s 28 . $.80,81$ 206 80, 84, 180, 206 $82,84,180$ (6) (a) 82 (b) .83 s 29 .80

(1) 80

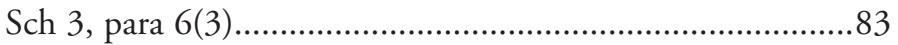

1991 Age of Legal Capacity (Scotland) Act (c 50).........................71

1992 Social Security Contributions and Benefits Act (c 4) ............89 s 137 .....

1995 Civil Evidence (Family Mediation) (Scotland) Act (c 6).....130 Children (Scotland) Act (c 36) ...... 25, 26, 27, 36, 37, 41, 42, $50,51,61,71,75,119,122,123,124$, $129,135,141,174,201,233,235$

Pt I $25,44,232$ Pt II 44,50 s 1(1). $42,43,50$

(a) (d)

(3) $.25,50$ s 2 121

(1)(a)-(d) 121

(c) 121

(2)(b) 233

(4) 121 s 3 206

(4) 168 
s 4 $75,76,77$

s 7 233

s 10 50

s 11 $24,25,26,28,36,37,43,50,77,119,120,121$, $122,129,135,136,167,168,215,232$ (1) $25,120,122$ (a) 120 (b) 120

(c) 120 (2). (d) 120

(b) 120 (i) 119 (d) 120 (e) $25,26,119,120,121,215$ 120,121

(5) $25,43,50,135$ (7) $25,39,135$ $25,39,50,121$ 44,45 (7D) $27,42,44,45$ s 15 164 s 16 121,168 .45

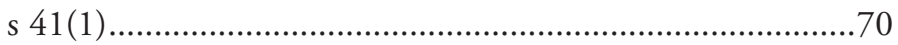

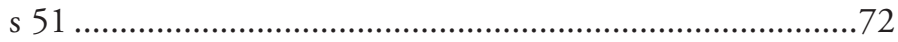

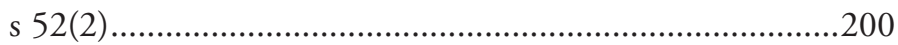

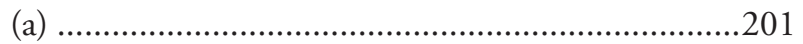

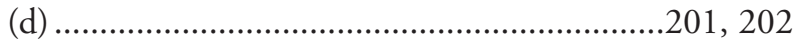

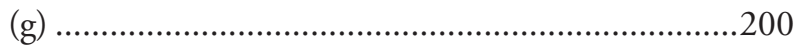

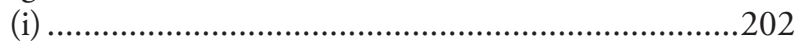

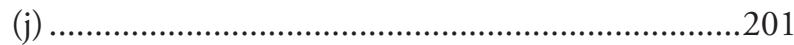

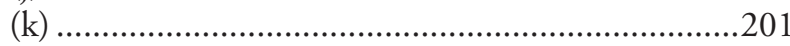

(m)

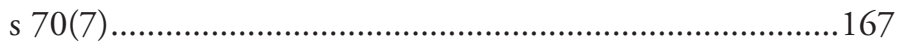

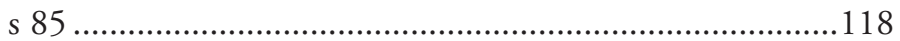

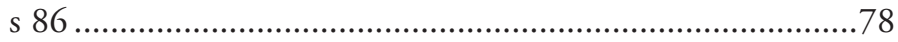


s $88(3)$ .78

s $93(2)$

Criminal Law (Consolidation) (Scotland) Act (c 39) .........217

Family Law Reform Act (Australia) ...................................51

1996 Education Act (c 56) .......................................................257

South African Constitution ........................................48, 49

s 8

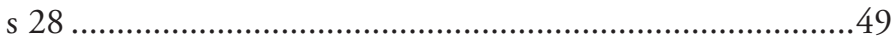

1997 Protection from Harassment Act (c 40)..............................260

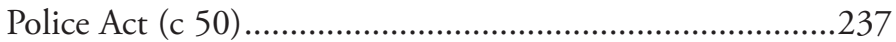

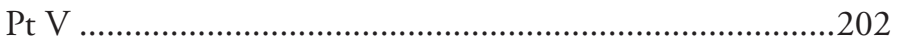

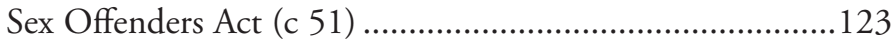

1998 Crime and Disorder Act (c 37) .......................................124

Human Rights Act (c 42)................... 45, 47, 77, 85, 86, 88

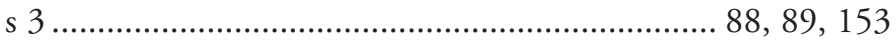

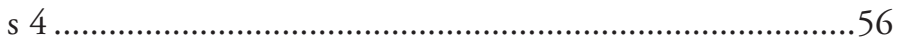

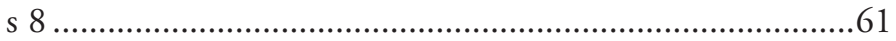

Scotland Act (c 46) ............................................................20

1999 Adoption (Intercountry Aspects) Act (c 18) ........... 19, 20, 22

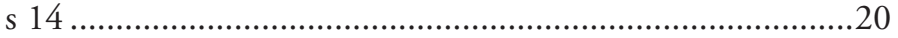

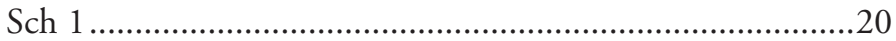

2001 Protection from Abuse (Scotland) Act (asp 14) .................260

2002 Adoption and Children Act (c 38) .................. 133, 135, 207

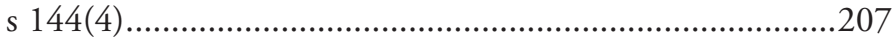

Washington Rev Code

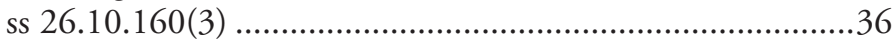

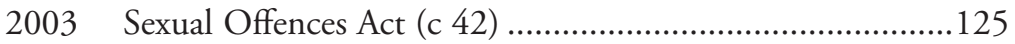

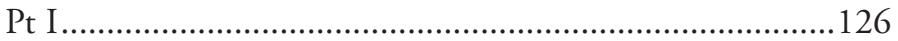

Pt II .............................................................................. 124

Protection of Children (Scotland) Act (asp 5) ....................124

Criminal Justice (Scotland) Act (asp 7) ..............................198

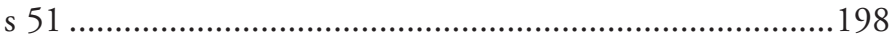

2004 Gender Recognition Act (c 7) ........... 56, 107, 114, 118, 179 Civil Partnership Act (c 33)....... 67, 107, 114, 130, 133, 145, $148,150,179,180,189,190,206,243,245,260$

s 87

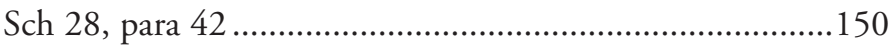

Education (Additional Support for Learning) (Scotland)

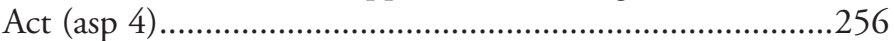

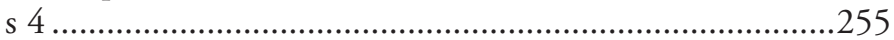

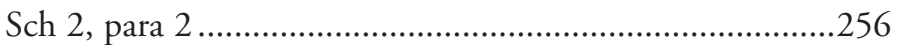

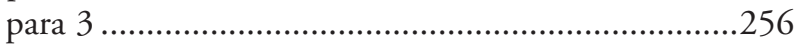


Antisocial Behaviour etc (Scotland) Act (asp 8)...123, 196, 201 s 12 201

2005 Protection of Children and Prevention of Sexual

Offences (Scotland) Act (asp 9) 126

2006 Children and Adoption Act (c 20) 164 Family Law (Scotland) Act (asp 2) $33,67,78,131,145$ $146,149,152,164,175,176,177,178$, $179,180,181,182,208,209,232,245,260$ s 2 101

s 3 32

s 15 147, 148

s 16 131,160

s 24 164

s 25 209

(2) 210 s 28 176, 178, 209, 212

(2) (b) 208 (3) (a) 176 s 35 149, 150, 152 152 151 (5) $.150,151$ s 38

Sch 2, para 2 .... 150

2007 Statistics and Registration Service Act (c 18) ......................189 s 69

Adoption and Children (Scotland) Act (asp 4)... 78, 107, 137 169, 180, 207 s $14(3)$ 166 s $29(3)$ 207 s 31 165 (3) (a) 165 (b) 166 (c) 166 (d) 166 166 166 166

s 89 168

s 90 168 
s $97(5)$ 168

s 103 ...... 167

Protection of Vulnerable Groups (Scotland) Act (asp 14)...202 2008 Human Fertilisation and Embryology Act (c 22) 84,107 182, 205, 206, 207 s 33 $84,207,208$

s 35 84, 206

s 36 206 ss $36-38$ .84

s 37 206

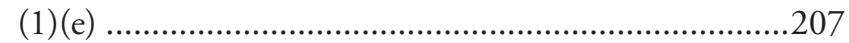
s 42 $164,206,207,208$ s 43 164, 206, 207 s 44 206

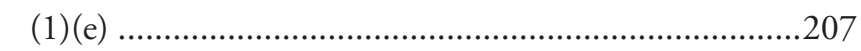
s $58(2)$...... 207

2009 Education (Additional Support for Learning) (Scotland)

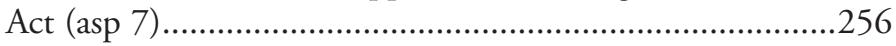
Sexual Offences (Scotland) Act (asp 9) ............. 126, 217, 246 2010 Equality Act (c 15) 193 Civil Partnership and Certain Rights and Obligations of Cohabitants Act (Republic of Ireland)

2011 Children's Hearings (Scotland) Act (asp 1) 73, 101, 169, 235, 259,262

s 79 235

s 160 235

s 187 241

s 200 235 Domestic Abuse (Scotland) Act (asp 13) .......... 259, 260, 262 s 3(2)..... 260 Forced Marriage etc (Protection and Jurisdiction) (Scotland) Act (asp 15). 261

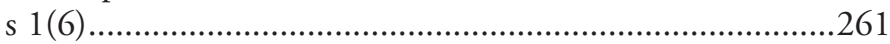

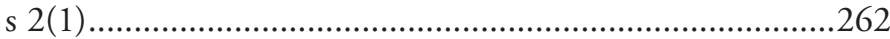

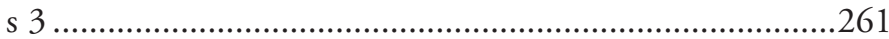

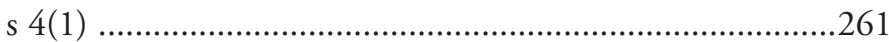

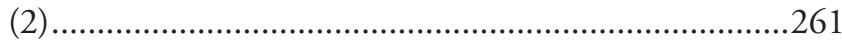

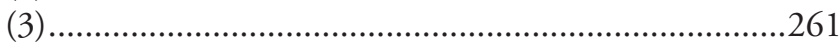


